American Journal of Immunology 7 (2): 24-28, 2011

ISSN 1553-619X

(C) 2011 Science Publications

\title{
Evaluating the Effect of Booster Dose of Hepatitis B Vaccine in Low-and Non-Responders Healthcare Workers and the Role of some Host-Related Factors
}

\author{
${ }^{1}$ Mojtaba Varshochi and ${ }^{2}$ Mehdi Haghdoost \\ ${ }^{1}$ Infectious Diseases and Tropical Medicine Research Center, Faculty Medicine, \\ ${ }^{2}$ Department of Infectious Diseases, Faculty of Medicine, \\ Tabriz University of Medical Sciences, Tabriz, Iran
}

\begin{abstract}
Problem statement: Hepatitis B comprises one of the major health problems worldwide. Health Care Workers (HCW) are a group at risk for Hepatitis B Virus (HBV) infection. Infection with hepatitis B virus has become a vaccine-preventable disease. Vaccination against Hepatitis B Virus infection (HBV) is safe and effective. The aim of this study is evaluation the immunologic response of booster dose of Hepatitis B vaccine in none and low responder health care workers and effects of some host-related factors. Approach: In a cross sectional descriptive analytic study carried out on the medical staff of Tabriz Shahid Madani Hospital in 2009-2010, we evaluated the immunologic response of booster dose of Hepatitis B vaccine in none and low responder health care workers and effects of some host-related factors. Results: Of 331 studied health care workers, 123 people (37.2\%) were male and 208 people $(62.8 \%)$ female. The mean antibody titer in the studied medical staff was $304.07 \pm 199.98 \mathrm{IU} \mathrm{L}^{-1}$ in the range of $0-1000$ and median of 330. Dividing the antibody titer into three groups of "no response" (Titer<10 IU L ${ }^{-1}$ ), "Low response" (Titer10-100 IU L ${ }^{-1}$ ) and "Good response" (Titer>100 $\mathrm{IU} \mathrm{L}^{-1}$ ) revealed that from 331 studied staff, 31 people $(9.4 \%)$ were in "no response" group, 40 people $(12.1 \%)$ in "Low response" group and 260 people $(78.59 \%)$ in "Good response" group and after one booster dose of vaccine in none and low responder group, 7 people $(2.1 \%)$ were in "no response" group, 5 people $(1.5 \%)$ in "Low response" group and 319 people (96.4\%) in "Good response" group. Conclusion: One booster dose of vaccine in people with low and none Response to hepatitis B vaccination cause to significantly increase of antibody titer so that, Good response rate increase from 78.5-96.4\% and low response rate decrease from $12.1-1.5 \%$ and none response rate decrease from $9.4-2.1 \%$. Use one booster dose of vaccine recommended in people with antibody titer blow 100 .
\end{abstract}

Key words: Hepatitis B vaccines, occupational diseases, prevention and control, immunization, vaccination, booster dose, Health Care Workers (HCW), host-related factors, medical staff, immunologic response

\section{INTRODUCTION}

Hepatitis B comprises one of the major health problems worldwide (Varshochi and Mahmodian, 2011; Perez et al., 1998). It may lead to chronic carrier state in 6-10\% of patients (Mandell et al., 2010) and chronic infection may result in various degrees of inflammation or necrosis, leading to cirrhosis and hepatocellular carcinoma. HCWs are at risk of $\mathrm{HBV}$ infection more than general population.

Hepatitis $\mathrm{B}$ vaccination is recommended for all Health Care Workers (HCW) at risk of exposure to infectious body fluids (Williams et al., 2001). Testing of blood for anti-HBs one month after vaccination is recommended to recognize non-responders as a booster dose will be beneficial in the majority of them (Perera et al., 2002).

Furthermore, post vaccination antibody testing should be restricted only to high-risk subjects (Zannolli and Morgese, 1997).

Some factor such as sex, age, obesity, route of injection and smoking can influence seroconversion rate (Wood et al., 1993; Mandell et al., 2010).

In our country, all neonates, HCWs, medical students, accidentally exposed peoples and other highrisk groups have vaccinated routinely against hepatitis $\mathrm{B}$, since 9 years ago.

Corresponding Author: Mojtaba Varshochi, Infectious Diseases and Tropical Medicine Research Center, Faculty of Medicine, Tabriz University of Medical Sciences, Tabriz, Iran Tel: +98-9143135846 
In antibody titer below 10, people become sensitive and despite having previous vaccination history, they may get infected by hepatitis B. therefore, serological examination of Hbs_ab and determination of its level in HCWs is necessary. In case of low antibody titer level, hepatitis B booster dose vaccination in inevitable.

The aim of this study is evaluation the immunologic response of booster dose of Hepatitis B vaccine in none and low responder health care workers and effects of some host-related factors.

\section{MATERIALS AND METHODS}

In a cross sectional descriptive analytic study carried out on the medical staff of Tabriz Shahid Madani Hospital in 2009-2010, we evaluated the immunologic response of the staff to vaccination against Hepatitis B and factors affecting it. In this study, HCWs, three were hepatitis B vaccine into the routine and the responses to 3 months after vaccination were studied. In our study, of 331 people, 260 of them had ideal Titer antibody titers, 40 and 31 of them had inadequate title and unacceptable antibody titer that one booster dose of hepatitis B vaccine injected for all of these and three to six months later, HBS antibody titer level was checked again in the same lab with the same kit and the effect of booster dose vaccination was examined in this group of people.

Medical staff is routinely vaccinated against hepatitis B and therefore antibody titration is essential to insure appropriate immunity.

The studied medical staffs are vaccinated by hepatitis B vaccine with fallowing properties (entitled EUVAX B, met the WHO requirements, made in Korea, LG life sciences company) for three doses $(0,1$ and 6 months) and antibody was titrated three months after vaccination. All utilized vaccines were from one brand and all tests were performed in one laboratory.

ELISA test was used to evaluate the samples used kit in this study was the Anti-HBs kit, made by ROCHE Company entitled "COBAS". Methods of this kit was ELISA, in which ELISA micro plates in this method ELISA micro plates are covered by antigen $S$ so that after adding serum containing antibodies against antigen S, antigens would bind with antibodies. In the next step, antigen $S$ conjugated with peroxides enzyme is added which attaches to the part of the antibodies not bound to antigens. Later, adding chromo gene and substrate dies the solution whose color can be read by ELISA reader.

After performing tests and reading the plates by ELISA reader, standard curves were provided using standard samples. Later using these curves, the concentration of antibodies in the tested sample were calculated. Based on the instructions of the kit manufacturer, antibody level less than 10 units per $\mathrm{mL}$ was considered negative and amounts higher as positive.

\section{RESULTS}

This study was carried out on 331 people from the medical staff and HBs_Ag titers were measured after three dose vaccination ( 0,1 and 6 months), the following results were obtained.

123 people $(37.2 \%)$ from the studied staff were male and 208 people $(62.8 \%)$ female. Demographic data of the studied medical staff are presented in Table 1.

The mean antibody titer in the studied medical staff was $304.07 \pm 199.98 \mathrm{IU} \mathrm{L}^{-1}$ in the range of $0-1000$ $\mathrm{IU} \mathrm{L}^{-1}$ and median of $330 \mathrm{IU} \mathrm{L}^{-1}$.

Antibody titer before and after booster dose of vaccine of the studied staff according to gender, smoking and hyperlipidemia are presented in Table 2. Repose to Vaccination in studied health workers at the base of smoking and hyperlipidemia are presented in Table 3. Repose to Vaccination in studied health workers with and without booster dose of vaccine was showed in Fig. 1. Titer of antibody of HCWs with and without booster dose of vaccine was showed in Fig. 2.

Table 1: demographic factors of health care workers

\begin{tabular}{lllr}
\hline & Sex & & \\
& Male & Female & P_Value \\
\hline Age (year) & $35 \pm 9$ & $31 \pm 6$ & $<0.001$ \\
Length (m) & $1.72 \pm 0.061$ & $0.59 \pm 0.05$ & $<0.001$ \\
Weight (Kg) & $73 \pm 12$ & $63 \pm 11$ & $<0.001$ \\
BMI & $25.65 \pm 4.12$ & $24.13 \pm 3.86$ & 0.002 \\
Smoker & 15 & 1 & $<0.001$ \\
HLP & 30 & 10 & $<0.001$ \\
\hline
\end{tabular}

Table 2: Antibody titer before and after booster dose of vaccine of the studied staff according to gender, smoking and hyperlipidemia

\begin{tabular}{llllll}
\hline & & $\begin{array}{l}\text { Titer antibody } \\
\text { without booster } \\
\text { dose (IU/L) }\end{array}$ & $\begin{array}{l}\text { Titer antibody } \\
\text { with booster } \\
\text { dose (IU/L) }\end{array}$ & \\
& & $\begin{array}{l}\text { Mean } \pm \text { Std } \\
\text { Deviation }\end{array}$ & P_V & $\begin{array}{l}\text { Mean } \pm \text { Std } \\
\text { Deviation }\end{array}$ & P_V \\
\hline Sex & Male & $340 \pm 205$ & 0.013 & $387 \pm 175$ & 0.137 \\
Smoking & Female & $283 \pm 195$ & & $359 \pm 164$ & \\
Hyperlipidemia & No & $307 \pm 199$ & 0.588 & $371 \pm 167$ & 0.501 \\
& Yes & $252 \pm 224$ & & $341 \pm 207$ & \\
& Yes & $318 \pm 214$ & 0.640 & $382 \pm 177$ & 0.617 \\
& No & $302 \pm 198$ & & $367 \pm 168$ & \\
\hline
\end{tabular}

Table 3: Repose to Vaccination in studied health workers at the base of smoking and hyperlipidemia

\begin{tabular}{lllllrrrr}
\hline & & \multicolumn{3}{c}{ Smoking } & & \multicolumn{3}{c}{ Hyperlipidemia } \\
& & No & Yes & P_V & Yes & No & P_V \\
\hline Titer antibody & Non responders & 27 & 4 & & 4 & 27 & \\
Without booster & Low responders & 37 & 3 & 0.020 & 5 & 35 & 0.859 \\
Dose (IU/L) & Good responders & 251 & 9 & & 31 & 229 & \\
Titer antibody & Non responders & 5 & 2 & $<0.001$ & 2 & 5 & 0.601 \\
With booster & Low responders & 3 & 2 & & & 5 & \\
Dose (IU/L) & Good responders & 307 & 12 & & 38 & 281 & \\
\hline
\end{tabular}




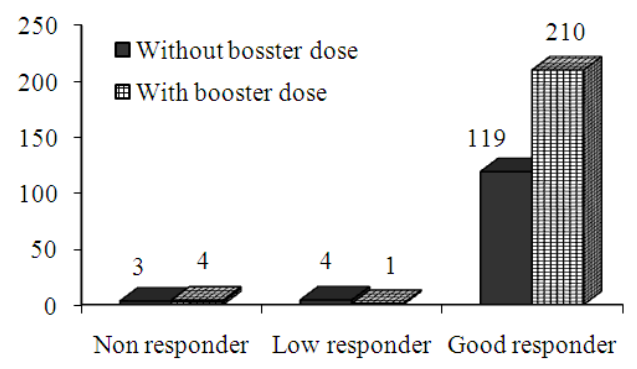

Fig. 1: Repose to vaccination in studied health workers with and without booster dose of vaccine

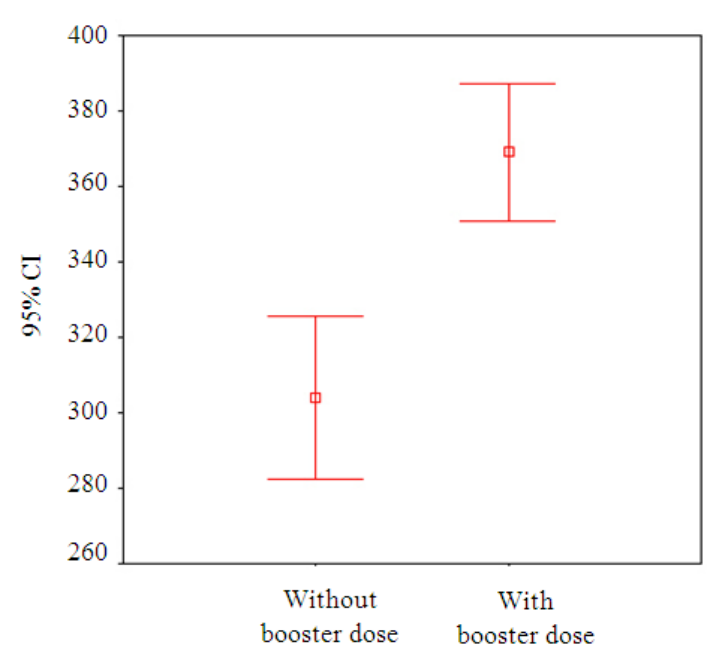

Fig. 2: Titer of antibody of HCWs with and without booster dose of vaccine

Dividing the antibody titer into three groups of "no response" (Titer $<10 \mathrm{IU} \mathrm{L}^{-1}$ ), "Low response" (Titer10$100 \mathrm{IU} \mathrm{L}^{-1}$ ) and "Good response" (Titer>100 IU L ${ }^{-1}$ ) revealed that from 331 studied staff, 31 people $(9.4 \%)$ were in "no response" group, 40 people $(12.1 \%)$ in "Low response" group and 260 people $(78.5 \%)$ in "Good response" group and after booster dose of vaccine, 7 people $(2.1 \%)$ were in "no response" group, 5 people $(1.5 \%)$ in "Low response" group and 319 people $(96.4 \%)$ in "Good response" group.

Response of the staff according to gender, smoking and hyperlipidemia wait and without booster dose of vaccine are presented in Table 2 and 3 which shows that response in the smoking staff was significantly less $\quad(\mathrm{p}<0.001)$ but no significant difference was observed in the response between genders $(\mathrm{p}=0.129)$ and no case of hyperlipidemia $(\mathrm{p}=$ 0.601 ) was reported.

There was a significant reverse linear relation between age and antibody titer in the studied staff and antibody titer decreased significantly as age increased $(\mathrm{p}=0.003, \mathrm{R}=-0.161)$ but no significant linear relation was observed between weight, height and BMI and antibody titer.

\section{DISCUSSION}

Use of the vaccine in high-risk health care professions has been modest, despite widespread participation in the establishment of vaccination programs among these institutions.

Postvaccination antibody testing and regular testing for antibodies is recommended only to high-risk subjects, especially to health care workers and subjects with immunodeficiency. In these cases, the booster dose should be administered in none responders and might include double doses (Alvarez et al., 2000). Although a high percentage of HCWs have been fully vaccinated with hepatitis $B$ vaccine, efforts need to be made to improve this coverage (Mahoney et al., 1997). Testing of blood for anti-HBs one month after vaccination is recommended to recognize nonresponders as a booster dose will be beneficial in the majority of them (Perera et al., 2000).

In the study of $2.5 \%$ also received the booster dose of the HBV vaccine (Duseja et al., 2002).

In our study of 331 HCWS, 71 (21.45\%) received booster dose of $\mathrm{HB}$ vaccine due to none or inadequate coverage.

In the study of Lok et al. (1988), age is considered as an effective factor in determining the response to the vaccine therefore highest response to the vaccine had achieved in the early patients and serum antibody titer had indirect correlation with age that good response rate was decreased from $86 \%$ in the fourth to $47 \%$ in sixth decade.

In our study, the antibody was reduced in patients with age but these changes were not significant.

In the studies of Shaw et al. (1989) and Minana (1996) the response rates in men were lower.

In our study, unlike the results of these studies, response rate in women was lower than men and mean of antibody titer was significantly higher in men than women.

In the study of Shaw et al. (1989) and Shapiro and Margolis (1992) immunological response in obese patients was low.

In our study, titer of antibody was reduced with increasing of weight and BMI, but these changes were not significant. 
Tomasiewicz et al. (1994) propose that timing of booster vaccination should be scheduled on the basis of anti-HBs level. It seems to be necessary to control the level of anti-HBs at least 3 years after the last dose of vaccination (Tomasiewicz et al., 1994).

Das et al. (2003) recommended that a single booster dose after 6 months in primary non-responders leads to good seroprotective anti-HBs antibody titers.

Although most participants responded to a booster dose of hepatitis B vaccine, the significance of the increased proportion of none responses among older adolescents might indicate waning immune memory(Samandari et al., 2007).

Although body mass index affected the response to the first hepatitis B booster, when full compliance to regular revaccination was ensured, all non- and lowresponders eventually reached sufficient anti-HBs levels (Clemens et al., 1997).

Overall, 73/76 (96\%) of students in the second group had protective concentrations of antibody after the booster dose (Bryan et al., 1992).

In our study, the ideal response to routine vaccination with three doses was $78.5 \%$ which increased to $96.4 \%$ with use a booster dose in the 71 people who had no acceptable response that this increase in response rate was significant.

\section{CONCLUSION}

Of 331 studied health care workers, 123 people $(37.2 \%)$ were male and 208 people $(62.8 \%)$ female. The mean antibody titer in the studied medical staff was $304.07 \pm 199.98 \mathrm{IU} \mathrm{L}^{-1}$ in the range of $0-1000$ and median of 330. Dividing the antibody titer into three groups of "no response" (Titer $<10 \mathrm{IU} \mathrm{L} \mathrm{L}^{-1}$ ), "Low response" (Titer10-100 IU L ${ }^{-1}$ ) and "Good response" (Titer>100 IU L ${ }^{-1}$ ) revealed that from 331 studied staff, 31 people $(9.4 \%)$ were in "no response" group, 40 people (12.1\%) in "Low response" group and 260 people (78.59\%) in "Good response" group and after one booster dose of vaccine in none and low responder group, 7 people $(2.1 \%)$ were in "no response" group, 5 people (1.5\%) in "Low response" group and 319 people (96.4\%) in "Good response" group. One booster dose of vaccine in people with low and none Response to hepatitis B vaccination cause to significantly increase of antibody titer so that, Good response rate increase from $78.5-96.4 \%$ and low response rate decrease from $12.1-1.5 \%$ and none response rate decrease from 9.4$2.1 \%$. Use one booster dose of vaccine recommended in people with antibody titer blow 100 .

\section{REFERENCES}

Alvarez, J.R.P., M.S.G. Holgado, J.L. Diaz and M.D. Rodriguez, 2000. Hepatitis B vaccination. Indications of the post-vaccine serologic test and booster doses. Rev. Esp. Salud Publica., 74: 475482. PMID: 11217237

Bryan, J.P., M.H. Sjogren, P. Macarthy, E. Cox and L.J. Legters et al., 1992. Persistence of antibody to hepatitis B surface antigen after low-dose, intradermal hepatitis B immunization and response to a booster dose. Vaccine, 10: 33-38. PMID: 1531719

Clemens, R., R. Sanger, J. Kruppenbacher, W. Hbel and W. Stanbury et al., 1997. Booster immunization of low- and non-responders after a standard three dose hepatitis B vaccine schedule-results of a post-marketing surveillance. Vaccine, 15: 349-352. PMID: 9141203

Das, K., R.K. Gupta, V. Kumar and P. Kar, 2003. Immunogenicity and reactogenicity of a recombinant hepatitis $\mathrm{B}$ vaccine in subjects over age of forty years and response of a booster dose among nonresponders. World J. Gastroenterol., 9: 1132-1134. PMID: 12717874

Duseja, A., L. Arora, B. Masih, H. Singh and A. Gupta et al., 2002. Hepatitis B and C virus-prevalence and prevention in health care workers. Trop. Gastroenterol., 23: 125-126. PMID: 12693154

Lok, A.S., C.L. Lai and P.C. Wu,1988. Prevalence of isolated antibody to hepatitis B core antigen in an area endemic for hepatitis B virus infection: Implications in hepatitis B vaccination programs. Hepatology, 8: 766-770. PMID: 2968945

Mahoney, F.J., K. Stewart, H. Hu, P. Coleman and M.J. Alter, 1997. Progress toward the elimination of hepatitis B virus transmission among health care workers in the United States. Arch. Intern. Med., 157: 2601-2605. PMID: 9531229

Mandell, G.L., J.E. Bennett and R. Dolin, 2010. Mandell, Douglas and Bennett's Principles and Practice of Infectious Diseases. 7th Edn., Churchill Livingstone, Philadelphia, ISBN: 0443068399, pp: 4028.

Minana, J.S. M.G. Ganuza, P.F. Millian and M.P. Fernnandez, 1996. Hepatitis B vaccine immunoresponsiveness in adolescents: A revaccination proposal after primary vaccination. Vaccine, 14: 103-106. PMID: 8852404 
Perera, J., B. Perera and S. Gamage, 2002. Seroconversion after hepatitis $\mathrm{B}$ vaccination in healthy young adults and the effect of a booster dose. Ceylon Med. J., 47: 6-8. PMID: 12001615

Perez, J.A.M., J.E.B. Santamarna, M.G. Luengo, L.C. Moreno and C.S. Alcazar et al., 1998. Coverage and immune response to hepatitis $\mathrm{B}$ vaccine in adolescents of the Guadalajara province. Rev. Esp. Salud Publica., 72: 119-126. PMID: 9643067

Samandari, T., A.E. Fiore, S. Negus, J.L. Williams and W. Kuhnert et al., 2007. Differences in response to a hepatitis B vaccine booster dose among Alaskan children and adolescents vaccinated during infancy. Pediatrics, 120: e373-e381. PMID: 17636112

Shapiro, C.N. and H.S. Margolis, 1992. Impact of hepatitis B virus infection on women and children. Infect. Dis. Clin. North Am., 6: 75-91. PMID: 1533649

Shaw, Jr., E.F. H.A. Guess, J.M. Roets, F.E. Mohr and P.J. Coleman et al., 1989. Effect of anatomic injection site, age and smoking on the immune response to hepatitis $\mathrm{B}$ vaccination. Vaccine, 7: 425-430. PMID: 2530717
Tomasiewicz, K., R. Modrzewska, A. Lyczak and G. Rzeszowska, 1994. Anti-Hbs level after the basic course of vaccination against hepatitis B in health care workers. Przegl Epidemiol., 48: 11-15. PMID: 8073126

Varshochi, M. and R. Mahmodian, 2011. Immunologic response to hepatitis $\mathrm{b}$ vaccine in health care workers: A screening program and evaluation of some host-related factors role. Am. J. Immunol., 7: 12-16. DOI: 10.3844/ajisp.2011.12.16

Williams, J.L., C.J. Christensen, B.J. McMahon, L.R. Bulkow and H.H. Cagle et al., 2001. Evaluation of the response to a booster dose of hepatitis B vaccine in previously immunized healthcare workers. Vaccine, 19: 4081-4085. PMID: 11427285

Wood, R.C., K.L. MacDonald, K.E. White, C.W. Hedberg and M. Hanson et al., 1993. Risk factors for lack of detectable antibody following hepatitis B vaccination of Minnesota health care workers. JAMA, 270: 2935-2939. PMID: 8254853

Zannolli, R. and G. Morgese, 1997. Hepatitis B vaccine: Current issues. Ann. Pharmacother., 31: 1059-1067. PMID: 9296247 